\title{
Qualitative research in primary care in Portugal: long overdue
}

John Yaphe*

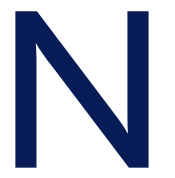

ot everything that is important can be counted and not everything that can be counted is important. This simple message is at the heart of qualitative research. What is really important to our patients, to our society and to our profession? If you want bread, go to the baker. If you want to answer these questions about health, disease and health care then ask the people involved. Patients, their families, professionals and stakeholders have been voicing their opinions on what things mean and how they work (or don't work) for a long time. We need ways to listen to them and understand their answers. This understanding of meaning and mechanisms is what qualitative research methods can give us.

A look at the index of this journal reveals a striking lack of qualitative studies conducted in primary care in Portugal. Perhaps they have been published elsewhere? A look at Medline shows that foreign publications of qualitative research conducted here in primary care are also lacking. Our colleagues in other professions in this country are teaching this topic and publishing the results of their qualitative research at home and abroad. We need to look at why this has happened to us and how this can be changed.

Qualitative research has been around since the beginning of science. When we first asked "What is this and how does it work?" we were already on the path. The scientific revolution of the $17^{\text {th }}$ century and the dominance of the quantitative scientific method launched us on another path. This was necessary for significant technological advances to occur. But the important questions in health were always there and needed answers provided by another approach. When a patient asks, "What does my disease mean to me and how can I make my life better?" we are in the qualitative domain. As Ian McWhinney tells us, the uniqueness of family medicine lies in combining scientific knowledge from

*Associate Professor, Community Health, School of Health Sciences, University of Minho evidence based medicine with our personal knowledge of individual patients. ${ }^{1}$ Personal knowledge is obtained through qualitative methods of observing, listening and reflecting.

The techniques of qualitative research are simple to understand. We talk to patients, we listen to their answers, and we ascribe meanings to these answers. We do this every day in every clinical encounter. What makes qualitative research different is that we do this in a standardized reproducible way to produce valid and reliable results.

There are three main techniques of qualitative research used in medicine. These are long interviews, focus groups and text analysis. The reader is referred to the excellent series of articles on qualitative research that appeared in the British Medical Journal in 1995 for a clear and enjoyable introduction to these methods. ${ }^{2}$

Long interviews as the name suggests are prolonged conversations with our subjects, usually patients or professionals. We allow people to talk freely about the subject that interests us, such as living with cancer or describing how examiners make judgements about students in oral examinations. ${ }^{3}$ We record this conversation and transcribe the text carefully. We then analyze the text looking for key phrases that illuminate our theme. Similar phrases are collected and grouped into categories. We try to find names for these categories to summarize our findings. We look for connections between categories to try to understand how the whole thing works. We do the same with the transcript obtained from the next patient, adding phrases to existing categories or creating new categories as they appear to us. We continue interviewing until no new categories appear and existing categories fill up with repeating phrases, saturating our categories. We look for confirming and conflicting evidence that give us as wide a range of views as possible. In our final report we explain our understanding of the phenomenon under study using illustrative examples as many direct quotes as possible. 
Focus groups are similar but we speak to a group of subjects all at once. This allows for group interaction. Members can support or challenge each other. This produces a wealth of observations in a very short time. The same rules of text analysis apply as in long interview studies. The meaning of a puzzling health practice in an immigrant community was unraveled in a focus group study. ${ }^{4}$

Text analysis on its own can be used when the data already exist such as in medical records or hospital discharge summaries. Filmed observations of patient behaviour can also be considered as a kind of text for analysis. The same process of creation of categories and an explanatory model applies here too. A study of videotapes of doctor patient communication revealed a model of dealing with conflict in the consultation. ${ }^{5}$

In order to put this on the Portuguese family medicine research agenda, several things need to change. First we need training in qualitative methods as part of our research training programs in medical schools, vocational training and continuing medical education. We need to collaborate with researchers from other disciplines (such as psychology, sociology, anthropology and nursing) who use these methods. We need to send interested candidates for specific training in this field. We need to try out these methods on the research questions that interest us in practice and submit our results for publication. This journal will certainly look favourably on submissions using qualitative methods. We can provide expert editing help to get these studies published.
Family medicine research in Portugal is taking great leaps forward in recent years. More family doctors are obtaining advanced degrees on the strength of their published research. Methods courses are taught in all universities and in all vocational training programs in the country. We can stimulate this development further by expanding our repertoire of research techniques. Qualitative research offers us a new perspective that can help us expand our knowledge and understanding. We look forward to seeing the results of your efforts in print on these pages.

\section{CONFLICT OF INTEREST \\ None}

\section{REFERENCES}

1. McWhinney IR. William Pickles Lecture 1996: The importance of being different. Br J Gen Pract 1996 Jul; 46 (408): 433-6.

2. Pope $C$, Mays N. Reaching the parts other methods cannot reach: an introduction to qualitative methods in health and health services research. BMJ 1995 Jul 1; 311 (6996): 42-5.

3. Yaphe J, Street S. How do examiners decide?: a qualitative study of the process of decision making in the oral examination component of the MRCGP examination. Med Educ 2003 Sep; 37 (9): 764-71.

4. Yaphe J, Schein M, Naveh P. Ethiopian parents' perception of their children's health: a focus group study of immigrants to Israel. Isr Med Assoc J 2001 Dec; 3 (12): 932-6.

5. Weingarten MA, Guttman N, Abramovitch H, Margalit RS, Roter D, Ziv $A$, et al. An anatomy of conflicts in primary care encounters: a multimethod study. Fam Pract 2010 Feb; 27 (1): 93-100.

ENDEREÇO PARA CORRESPONDÊNCIA

yonahyaphe@hotmail.com 